\title{
VERKLEBUNG VON LEBERZELLBALKEN BEI SÄUGETIEREN
}

VON

\author{
Dr. A. B. DROOGLEEVER FORTUYN. \\ (Anatomisch Kabinet, Leiden)
}

(Mit 3 Figuren im Tekst)

Das Verständnis des mikroskopischen Baues der Säugetierleber hat grosse Schwierigkeiten mit sich geführt schon von Anfang der Zeit an, wo die Technik weit genug fortgeschritten war um sich mit diesem Thema einzulassen. Die Art der Gallenkapillaren, die Struktur der Blutkapillaren, die Verbreitung der Lymphgefässe sind Probleme, die heute teils gelöst, teils noch Streitobjekte sind, die uns hier aber nicht beschäftigen sollen. In diesem Artikel soll nur auf die gegenseitige Lage von Leberzellen, Gallenkapillaren und Blutkapillaren geachtet werden.

HERING (1867) ist wohl der erste gewesen, der bezüglich dieser Lage sich eine Vorstellung machte, die deutlich genug war, auch bei anderen, ja, selbst jahrelang allgemein Eingang zu finden. Diese Vorstellung wurde in einem Schema das BRAus (1896, Fig. 1) publizierte und das auch in dem Handbuch von OpPel (1900, Fig. 559) zu finden ist, in Bild gebracht, aber vor dieser Zeit wurde es scheinbar schon lange auf Vorlesungen an verschiedenen Universitäten den Studenten vorgezeichnet. Hering selbst (1867) gibt nur einen Teil dieses Schemas. Den Auffassungen HeRING's von dem Bau der Säugetierleber haften eine Menge ernste Fehler an, selbst wenn mann berücksichtigt, dass denselben im Besonderen der Bau der Nagetierleber zu Grunde liegt. BRaus (1896) hat in einer aussergewöhnlich schönen Untersuchung, bei welcher auch niedere Vertebraten studiert wurden, diese Fehler grösstenteils ans Licht gebracht. Nach seiner Ansicht besteht die Leber der Vertebraten aus einem Netzwerk von mit einander anastomosierenden, aus Leberzellen bestehenden Leberröhrchen (bei Säugetieren, bei denen sie im Querdurchschnitt aus zwei Zellen bestehen Leberzellbalken genannt) deren Lumina die Gallenkapillaren sind und die mit einem Netzwerk, von Blutkapillaren durcheinander verschlungen liegen. (Vergl. Fig. 3). Braus unterschied unter den Maschen des Netzwerks der Gallenkapillaren 'vasozonale und cytozonale Maschen. Im ersten Falle umschliesst ein Ring Gallenkapillaren eine Blutkapillare und ist dieser also gefässbegrenzend, vasozonal; im zweiten Fall ist die Masche enger zugezogen und umschliesst nur eine oder mehrere Leberzellen, sodass sie zellbegrenzend oder cytozonal ist. Der rein tubulöse Charakter der Leber ist dann hiermit schon verloren gegangen. Es gibt Tiere, wie der Aal, deren Leber beinahe ausschliesslich vasozonaie Maschen enthält und dadurch lose gebaut ist, sodass die Struktur nicht schwer zu begreifen ist. Aber es gibt andere, bei denen das Vorkommen vieler cytozonaler Maschen der Leber einen compakteren, schwer zu entwirrenden Bau verleiht. Die Säugetiere im allgemeinen und unter ihnen hauptsächlich die Nagetiere gehören zu dieser Gruppe, es kommt zwar niemals so weit, dass, wie im Schema von HeRING, alle Maschen von Gallenkapillaren cytozonal sind.

Wenn in den Säugetierlebern mit den cytozonalen und vasozonalen Maschen der Gallenkapillaren und Leberzellbalken nichts besonders der Fall wäre würde jede Leberzelle in Durchschnitten, in denen die. dazu gehörige Gallenkapillare quer getroffen war, höchstens an zwei Blutkapillaren (bei cytozonalen Maschen) grenzen können. Das ist nun nicht der Fall. In dem Leberschema HerinG's grenzt jede Leberzelle an vier Blutkapillaren. Auch das ist, selbst bei Nagetieren, gewiss nicht immer wahr, 
doch es kommt wohl wiederholt vor, dass eine Leberzelle, die zu einem quer durchgeschittenen Leberzellbalken gehört, mit zwei, drei oder vier Blutkapillaren in Berührung ist.

Diese Erscheinung war Braus sehr gut bekannt, aber sie wird nur teilweise durch das Dasein von cytozonalen Maschen erklärt und ich habe weder bei ihm, noch sonst irgend wo in der Literatur die ergänzende Erklärung finden können. Ich glaube, dass die Erklärung in der Tatsache gesucht werden muss, dass die Leberzellbalken der Säugetiere nicht nur netzförmig anastomosieren, aber ausserdem mit einander verklebt sein können. In der Fläche, in der die Leberzellbalken mit einander verklebt sind, kann natürlich keine Gallenkapillare laufen wie im Zentrum des Leberzellbalkens. Denkt man sich alle Verklebungen losgemacht, dann muss man eine reine Reihe von anastomosierenden Leberzellbalken überbehalten, worin jede Leberzelle höchstens mit einer Blutkapillare in Berührung steht. Die unparteiischste Weise dies alles zu erklären, ist vielleicht die, dass ich dazu eine Figur Braus' (1896, Taf. 31, Fig. 67) wähle. Es ist (Fig. 1) eine Abbildung eines Leberschnittes von Mus musculus, worin, wie

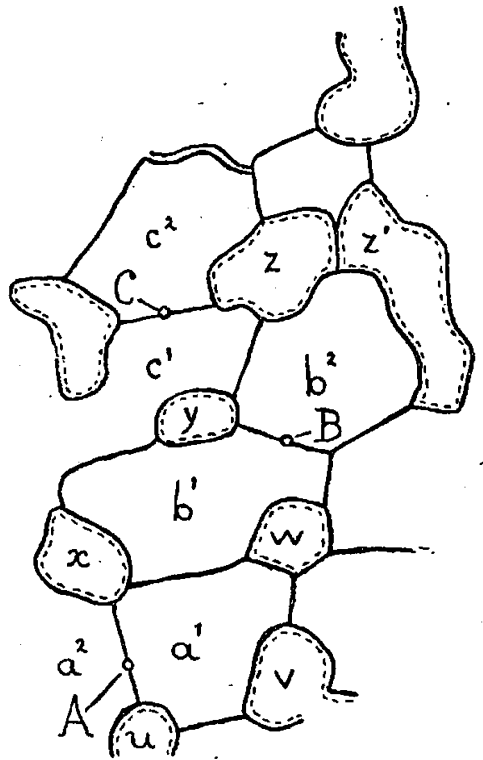

Fig. 1.

Leberschnitt von Mus musculus. Nach BRAUs (1896, Taf. 31, Fig. 67).

$\mathrm{A}, \mathrm{B}, \mathrm{C}=$ Gallenkapillaren:

$a^{1}, a^{2}, b^{1}, b^{2}, c^{1}, c^{2}=$ zugehörige Leberzellen.

$\mathrm{u}$ bis $\mathrm{z}=$ Blutkapillaren.

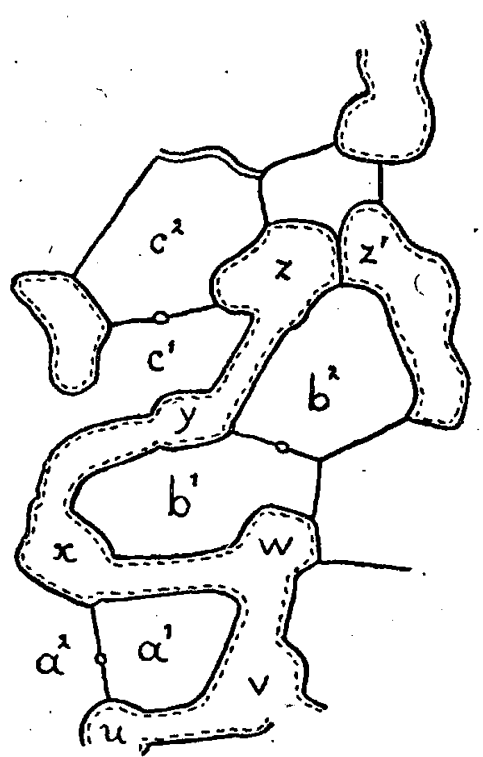

Fig. 2.

Dieselbe Figur wie Fig. 1 aber die Verklebungen der Leberzellbalken sind aufgehoben.

BRAUS selbst bemerkt, die unterste Leberzelle an vier Seiten mit Blutkapillaren in Berührung steht. Diese Zelle, $a^{1}$, bildet die Hälfte eines quer durchgeschnittenen Leberzellbalkens, dessen andere Hälfte von der nur teilweise sichtbaren Zelle $\mathrm{a}^{2}$ gebildet wird. Man sieht dieses von der quer durchgeschnittenen Gallenkapillare, A, zwischen den beiden. Ueber dieser ersten Gallenkapillare A sieht man noch eine zweite $B$ und eine dritte $C$ quer durchgeschnitten mit den dazu gehörigen Leberzellen $b^{1}, b^{2}, c^{b}$ und $c^{2}$. Zwischen den Leberzellbalken sieht man eine Anzahl Blutkapillaren, deren Wand ich durch eine punktierte Linie, andeute und von denen sich sechs mit den Buchstaben $u$ bis $z$ angab. In der Figur sieht man zwischen $u$ und $v, v$ und $w, w$ und $x, x$ und $y, y$ und $z$ Grenzen zwischen zwei Leberzellen, die keine Gallenkapillaren enthalten und also, weil wir es hier mit quer durchgeschnittenen Leberzellbalken zu tun haben, als Verklebungen von benachbarten Leberzellbalken, u. a. A mit B und $B$ mit $C$ aufgefasst werden müssen. (Bei dem Längsdurchschnitt eines Leberzellbalkens findet man natürlich Zellen von ein und demselben Leberzellbalken, die ebensowenig Gallenkapillaren zwischen sich enthalten, aber dieser Fall liegt hier nicht vor). Denkt man sich, sowie es in Fig. 2 vorgestellt ist, die Verklebungen aufgehoben und also die Blutkapillaren $\mathrm{u}, \mathrm{v}, \mathrm{w}, \mathrm{x}, \mathrm{y}$ und $\mathrm{z}$ in einander fliessend, dann zeigt sich, sowie aus den Regeln BRAus' folgen müsste, Zelle $\mathrm{a}^{1}$ anstatt mit vier mit einer Blutkapillare in Berührung, Zelle $b^{1}$ mit einer anstatt mit drei, Zelle $b^{2}$ mit zwei anstatt mit drei und Zelle $c^{1}$ mit zwei anstatt mit drei in Berührung. Zelle $b^{2}$ würde mit einer Kapillare in Berührung sein, wenn diese sich nicht scheinbar gerade verzweigt hätte; $z$ und $z^{\prime}$ sind nicht durch eine Leberzelle, 
doch durch eine doppelte Gefässwand getrennt und dürfen deshalb als eins gerechnet werden. Zelle $c^{1}$ würde wahrscheinlich mit nur einer Blutkapillare in Berührung zu bringen sein, wenn diese Zelle von Braus vollständig gezeichnet worden wäre. Man könnte sagen, die Gallenkapillaren A und B und auch $B$ und $C$ könnten zu einer cytozonalen Masche gehören, sodass die Verklebung von $a^{\prime}$ mit $\mathrm{b}^{1}$ und von $b^{2}$ mit $c^{1}$ dadurch schon durch BRAus erklärt sei. Die anderen Verklebungen bleiben dan jedoch unerklärt und es sind gerade diese auf welche ich die Aufmerksamkeit lenken möchte.

Aus der Analyse von Fig. 1 zeigt sich also, dass die Tatsache, dass eine Leberzelle auf Querdurchschnitt mit mehr als einer Blutkapillare in Berührung sein kann, durch Verklebung benachbarter Leberzellbalken erklärt werden muss, nicht allein in cytozonalen Maschen sondern auch ausserhalb solcher. Die Verklebungsflächen sind an dem Fehlen von Gallenkapillaren in ihrem Verlauf zu erkennen. Ich habe an Leberpräparaten von Mensch und Säugetieren verschiedentlich dasselbe konstatieren können.

Dass die Leberzellbalken der Säugetiere verklebt sein können, folgt auch wohl direkt aus der Ontogenie der Leber. Entsteht diese doch, wie bekannt, wie ein solider Zellenklumpen, worin die Blutgefässe hineinwachsen und die Leberzellen durch die Blutkapillaren stets mehr aus einander gedrungen. werden, was so zu verstehen ist, dass sie mindestens als ein Netzwerk von Leberzellbalken zusammenhängen bleiben. Geht dieses Auseinanderdringen nicht so weit, wie es gehen könnte, so bleiben Verklebungen zwischen den Leberzellbalken bestehen. Fig. 3 kann dies vielleicht noch näher

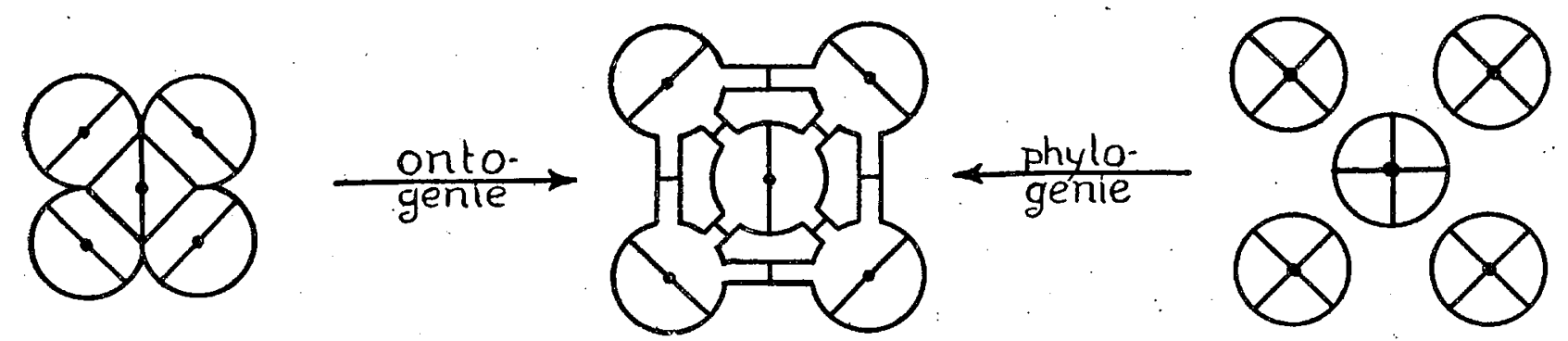

Fig. 3.

Schematische Darstellung der ontogenetische und phylogenetische Bildung der Verklebungen der Leberzellbalken. Links: fünf quer durchgeschnittene Leberzellbalken einer embryonalen Säugetierleber. Es sind noch keine Blutkapillaren dazwischen hineingewachsen.

In der Mitte: fünf quer durchgeschnittene Leberzellbalken einer erwachsenen Säugetierleber mit gegenseitigen Verklebungen und mit Blutkapillaren zwischen sich. Die Zellen des mittleren Leberzellbalkens grenzen je an drei Blutkapillaren.

Rechts: fünf quer durchgeschnittene Leberröhrchen eines erwachsenen niederen Vertebraten ganz von einander getrennt durch Blutkapillaren.

erklären. Dieser ontogenetische Prozess ist dann jedoch gerade das umgekehrte von dem, was sich phylogenetisch in der Leber abgespielt hat. Phylogenetisch ist ja, wie aus den Untersuchungen Braus' und anderer gezeigt, die Leber, ursprünglich eine rein tubulöse Drüse, in der alle Leberröhrchen frei von einander waren, zu einem Organ geworden, dessen tubulöser Bau nur mit grösster Mühe zu erkennen ist durch die vielen Verwachsungen und Verklebungen, die zwischen den Leberröhrchen aufgetreten sind (Fig. 3).

Es sei nebenbei noch bemerkt, dass wir hier wieder ein Beispiel haben von einem Fall, der im Gegensatz steht zu dem biogenetischen Grundgesetz von HAECKEL, das ja immer mehr an Grund zu verlieren scheint.

\section{LITERATUR.}

BRAUS (1896), Untersuchungen zur vergleichenden Histologie der Leber der Wirbelthiere, Denkschriften der medicinischnaturwissenschaftlichen Gesellschaft zu Jena, Bd. 5, 1894-97.

HeRING (1867), Ueber den Bau der Wirbeltierleber, Archiv für mikroskopische Anatomie, Bd. 3, 1867.

OPPEL (1900), Lehrbuch der vergleichenden mikroskopischen Anatomie, Bd. 3, 1900. 